\title{
LOGISTICS SECURITY MONITORING AND REAL-TIME TRACKING BASED ON RFID TECHNOLOGY -DESIGN AND APPLICATION OF ELECTRONIC SEAL Yu Shao
}

\author{
Department of Computer Science, Ningbo Dahongying University, Ningbo, 315175, China \\ dhyshaoyu@163.com
}

Keywords: RFID technology; Container transportation; Electronic seal

\begin{abstract}
For the opacity in the process of container road transportation and security problems, this essay introduces a reusable electronic seal, logistics safety monitoring and real-time tracking system based on RFID technology, which can effectively reduce or avoid the losses brought about by the abnormal events, and provide evidence for event traceability.
\end{abstract}

\section{Introduction}

RFID (Radio Frequency Identification) is a non-contact automatic identification communication technology aroused in the 1990s. It utilizes radio frequency signals by space coupling (alternating magnetic field or electromagnetic field) to realize non-contact information transmission and achieve recognition through the transmission of information [1]. It has a large number of advantages such as high speed, large storage space, strong penetrability, long service life, waterproof, antimagnetic, high temperature resistant and so on [2].

In terms of container intelligent applications, the United States first applied RFID technology to container management, and has a global container shipping tracking service system, and the harbor Chad, South Carolina is the last city to apply it in 2007. For domestic study on container management system, Rizzo and Marcello Barboni proposes a RFID system based on active identification to improve container transportation and supply chain security [3].Magalie Laniel and Jean - Pierre Emond utilized the three frequency band RFID reader to detect electronic label signal intensity, so as to realize the real-time temperature tracking and improve the management of perishable things [4].Some experts believe that the significance of the application of RFID technology in container transportation management is to not only break the bottleneck of information asymmetry of container transportation, and low collection efficiency, but also bring a revolution that will sweep the global logistics industry with the expanding application of the technology [5].

The purpose of this article aims at effectively applying automatic identification technology and transmission switching technology to the goods and vehicle tracking so as to realize the real-time location of container vehicle positioning and real-time condition monitoring, etc. As a result, the efficiency and effectiveness of the logistics are greatly improved.

\section{Structure of the system}

This system includes electronic seal, monitor client, vehicular communication terminal and the back-office server system. A number of electronic seal can make data exchange via RFID wireless communication and vehicular communication terminal. Vehicular communication terminal can make wireless data exchange with back-office server system through GPRS/CDMA/ WIFI / 3 G wireless data exchange and back-office server system and back-office server system can make data exchange through the Internet and monitor client. The structure of the system is shown in Figure 1. 


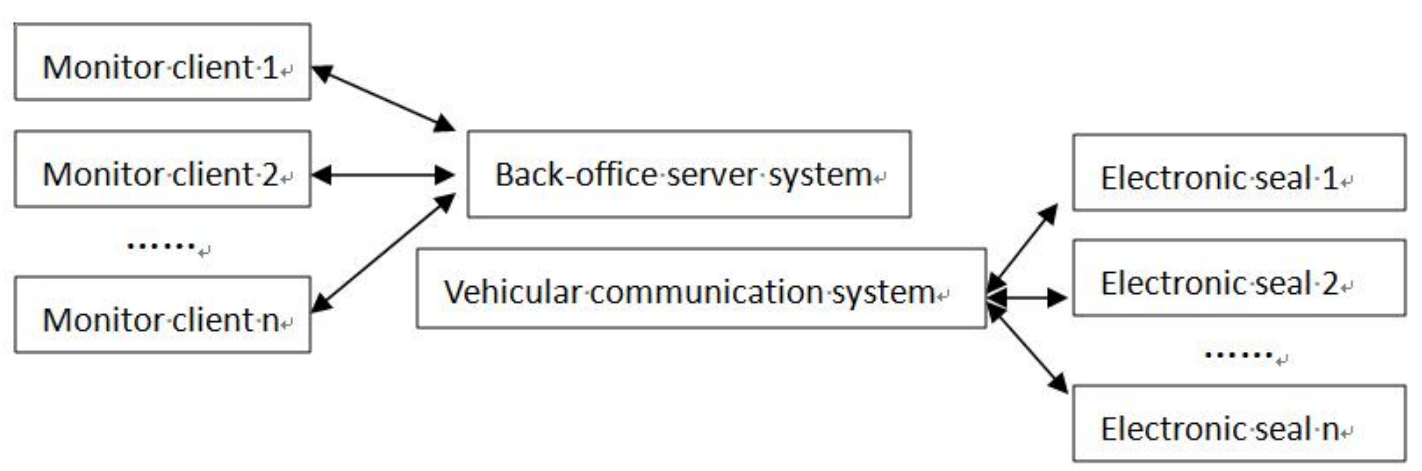

Fig.1. Structure of the system

\section{Structure of system hardware}

System hardware is made up of three parts: electronic seal, electronic seal reader as well as GPS vehicular communication terminal. As core part of the system, electronic seal bind the information (such as sealing containers, illegal open of the alarm, etc.) with vehicle mobile through the corresponding electronic seal reader, and make real-time transmission to the back server system. Electronic seal reader is based on the active RFID electronic seal reading equipment, connected with GRPS car vehicles, and a back server operation is not needed in the process of the electronic seal and the reader real-time binding.GPS vehicular communication terminal is a GPS device with GPS satellite positioning and communication functions, which can send own GPS location information to the designated back server system by the specific methods of communication. The hardware topology structure and physical structure of the system is shown in Figure 2.

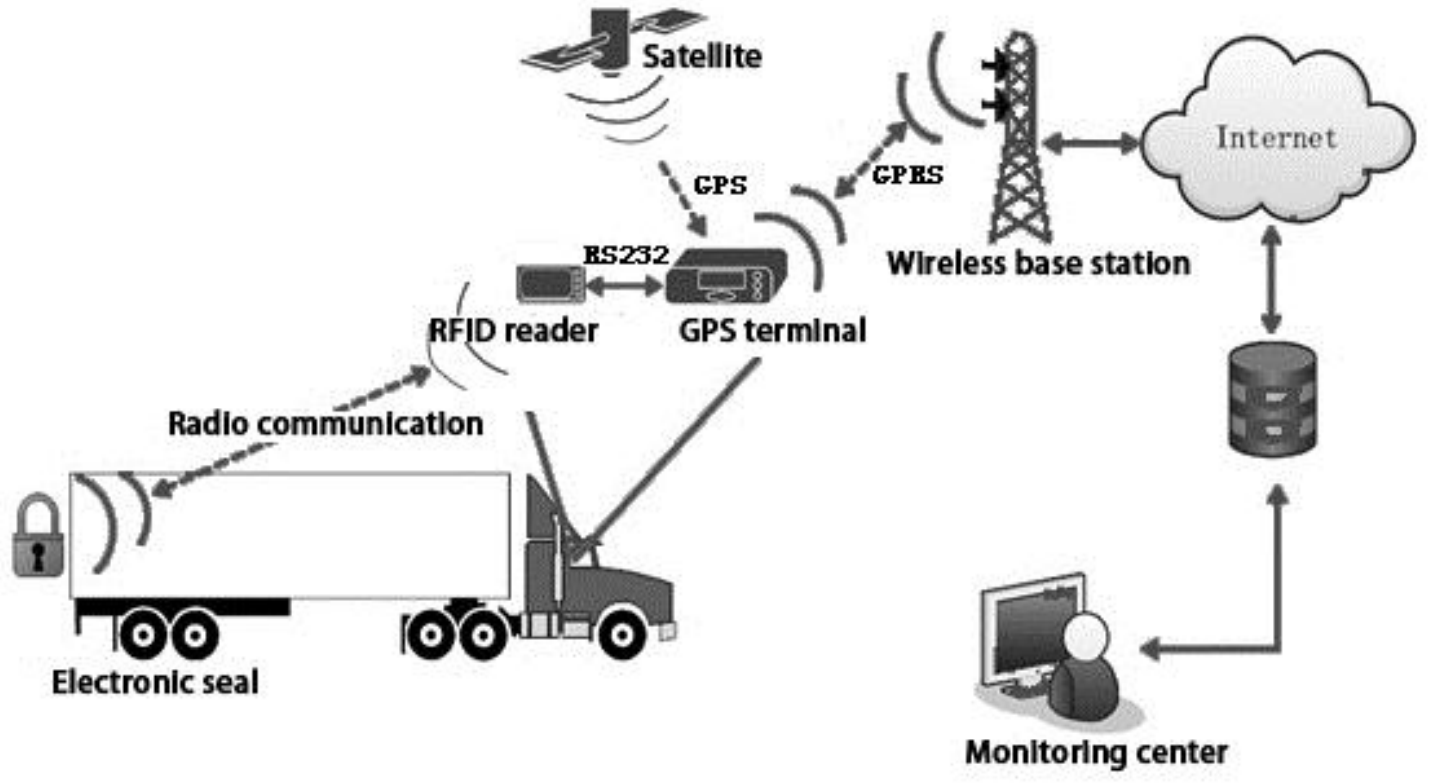

\section{Electronic Seal}

Fig. 2. Topology structure of system hardware

Electronic seal, employing RFID technology, has a unique identification number, with which you can store the user information such as vehicles, containers. On the basis of traditional mechanical seal function and working process, it has small volume, light weight, high scalability, high interference capability, reliability, security, convenient operation and many other advantages. It consists of a lock body and lock rod, whose internal composition and the working process is as follows: 
(1) The lock body contains double lock hole and lock core, LED lamp, storage, RFID communication module, test device, the external trigger device, battery and microprocessor segments.

(2) The lock rod is installed on the vehicle crate arm lock, and the electronic seal and crate locking arm are locked through lock hole and lock core. The lock rod is disposable and it will directly be broken away from below the lock hole when unlocked. Lock body will be recycled when unlocked and it can be reused after reset by triggering with external trigger device.

(3) The LED indicator light of lock body, the memory, RFID communication module, test device, external trigger device are connected with the microprocessor. RFID communication module connects the first antenna, and vehicle communication equipment connects the second antenna. RFID communication module communicates with vehicle communication equipment through the first antenna and the second antenna.

Microprocessor model inside the lock body is MSP430F2272, and which is an ultra-low power consumption of mixed signal processors of TI (Texas instruments) with the built-in two 16-bit timers, 10 bit A/D converter and DTC (data transfer controller), two general operational amplifier, a serial communication interface, and $32 \mathrm{I} / \mathrm{O}$ pins. Of course, other microprocessors with the same function can also be used.

\section{GPS vehicle-mounted communication terminal}

Vehicle communication terminal consists of GPRS/CDMA/WIFI / 3G data transfer module, data storage module, real-time clock module, GPS module, LCD screen, button, traffic information detection module, RFID communication module, a microprocessor and power management module, among which GPS module and RFID communication module are most important.. GPS module is responsible for the connection of GPS signals, and RFID communication module is responsible for communication with back-office system, the module block diagram is shown in Figure 3. Among them, the GPS module is connected with the first antenna and the data transmission module is connected with the third antenna, and RFID communication module is connected with the second antenna. Data transmission is made through the third antenna module and back-office server system, and communication will be made through the second antenna RFID communication module and electronic seal devices.

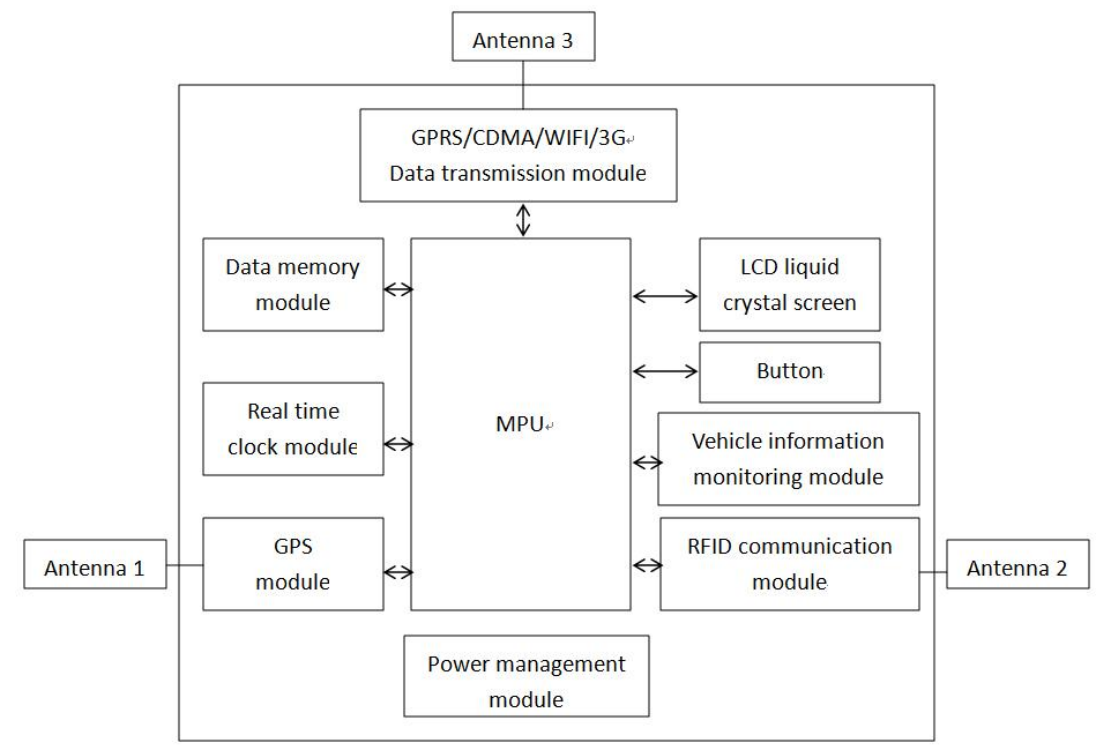

Fig.3. Diagram of vehicle communication terminal module

The microprocessor of vehicular communication terminal adopts LPC1766, which is a highly elaborated 32-bit embedded processor of NXP (Enzhipu semiconductor company). It is 
widely applied to communication, control, security and other fields. Of course, you can also use other microprocessor with the same function.

\section{Structure of system software}

System software is required to provide function of monitoring for a specific vehicle, containers and information processing. Software system receives related information about electronic seal and makes the corresponding processing by sending the control commands to the vehicle communication equipment, monitoring the mobile container vehicle. The system can also match the processed vehicle' information with electronic map so that the exact location of the vehicle is shown in the map. Furthermore, the moving trajectory of the vehicle can be replayed and queried, making the tracking of a moving vehicle. In addition, the system sets up the user's permissions in order to reduce the damage caused by wrong operation of the system [6].

The system combines $\mathrm{B} / \mathrm{S}$ structure and $\mathrm{C} / \mathrm{S}$ structure, giving priority to $\mathrm{B} / \mathrm{S}$ structure with auxiliary $\mathrm{C} / \mathrm{S}$ structure. B/S structure is used for data transmission in a network, such as vehicle information query, update, etc. With the integrated development of Microsoft Visual Studio.NET, the.net Framework can develop Windows application program on the basis of C/S structure and Web application program based on B/S structure.

Function modules of the system include six modules: electronic seal vehicle list module, GIS monitoring function module, track replaying function module, early warning management function module, administrator function module and system management function module.

\section{Remarkable achievements of the system}

\section{Quick tracking query}

The time, place, vehicles, and information about containers and logistics operation which are stored in the RFID tags can realize real-time tracking and full monitoring and queries. In the whole logistics links, the RFID reader will be installed in the appropriate location to make real-time tracking of vehicles and goods location, status, and its transport track at any time by online monitoring client-side - electronic seal monitoring platform. At the same time, if relevant early warning situations occur, such as system exception, successful sealing, battery alarm, seal failure alarm, GPS check alarm, etc., information can be immediately sent to the back-office server, and the administrator will be notified and disposal it.

\section{High safety of the goods}

Real-time monitoring of container with RFID technology solves the problems of stolen or lost containers and huge economic losses are recouped. The electronic seal monitoring platform can real-time monitor when and where to open it illegally, and when the alarm message can be sent to the back-office server when the electronic seal is opened in abnormal cases. At the same time, when these abnormal incidents happen, effective evidence will be provided by querying the information stored in the internal RFID tags of the electronic seal.

\section{Promotion of the service quality}

All the nodes in the supply chain of the containers such as the owner, freight forwarding, warehousing, cargo handling, insurance companies, transportation companies, banks and other users can access to the system through the electronic seal monitoring platform. In terms of supply chain management, standardized management and comprehensive monitoring and be made in the whole container logistics system through the platform.

\section{Conclusion}


According to the situation of current logistics security monitoring and real-time tracking, radio frequency identification technology better than bar code technology and logistics information system based on the technology are put forward. As to the current container security problems in the logistics transportation, a new kind of electronic seal based on RFID technology is designed with the study of RFID technology. It can solve the problems which often occur in the traditional seal during the process of transportation such as easy to open, copy and forgery, low security and customs clearance efficiency. What's more, the monitoring platform of electronic seal is designed to realize the safety monitoring and real-time tracking of logistics. The real vehicle test is carried on after the completion of the system development, and the test results show that the system can basically meet the design requirements, which realizes the expected goal.

\section{Acknowledgement}

In this paper, the research was sponsored by the scientific research fundation of Dahongying University (Project No. 1320141003).

\section{References}

[1] Xiaoguang Zhou \& Xiaohua Wang. Principles and Application Examples of Radio Frequency Identification (RFID) Technology. Beijing: Post and Telecom Press, 2006.11.

[2] Daiyou Jiang. System Architecture and Key Techniques of IOT and Related Problems. Journal of Electronic Design Engineering, 2012, 20 (4):143-145.

[3] Francesco Rizzo, Marcello Barboni, Lorenzo Faggion, Graziano Azzalin, Marco Sironi. Improved Security for Ltd. Transports Using an Innovative Active RFID System. Journal of Network and Computer Applications, 2010.

[4] Magalie Laniel, Jean - Pierre Emond,, Ahmet, E Altunbas, RFID Behaviors Study in Enclosed Marine Container for Real Time Temperature Tracking. Sens \& Instrumen Food Qual, 2009.3:34-40.

[5] Jia Zhang. A Study on Container Transportation Management Model Based on RFID [D]. Master's degree thesis, Nanjing University of Aeronautics and Astronautics. 2008, 3:36 - 38.

[6] Wenhui Chen. A Study on Postal Communication Transportation Management System Based on GPS/GPRS. Master's degree thesis, Xi 'an University of Electronic Science and Technology, 2010, $10: 42$. 